\title{
De niños a maestros: la iniciación de los artistas de las danzas rituales ayacuchanas
}

D'enfants à maîtres : l'initiation des artistes des danses rituelles d'Ayacucho From child to maestro: artists initiated into Ayacucho ritual dances

Jeanne Saint-Sardos

\section{OpenEdition}

Journals

Edición electrónica

URL: http://journals.openedition.org/bifea/4220

DOI: $10.4000 /$ bifea.4220

ISSN: 2076-5827

Editor

Institut Français d'Études Andines

Edición impresa

Fecha de publicación: 1 diciembre 2013

Paginación: 473-489

ISSN: 0303-7495

Referencia electrónica

Jeanne Saint-Sardos, « De niños a maestros: la iniciación de los artistas de las danzas rituales ayacuchanas », Bulletin de l'Institut français d'études andines [En línea], 42 (3) | 2013, Publicado el 08 diciembre 2013, consultado el 05 noviembre 2020. URL : http://journals.openedition.org/bifea/4220 ; DOI : https://doi.org/10.4000/bifea.4220

\section{c) $(1) \ominus$}

Les contenus du Bulletin de l'Institut français d'études andines sont mis à disposition selon les termes de la licence Creative Commons Attribution - Pas d'Utilisation Commerciale - Pas de Modification 4.0 International. 


\title{
De niños a maestros: la iniciación de los artistas de las danzas rituales ayacuchanas
}

\author{
Jeanne Saint-Sardos*
}

\section{Resumen}

Los artistas de las danzas rituales de competencia de Ayacucho (Perú) empiezan desde jóvenes y a veces muy niños a aprender su arte. Que quieran ser músicos, danzantes de tijeras, huaylías, pastores o negritos de cinta, que vivan en la Sierra o en Lima, que aprendan de una u otra forma, todos tienen no solamente que aprender a dominar las técnicas instrumentales o corporales pero también asimilar perfectamente las reglas socioreligiosas como los usos y costumbres. Efectivamente, después de unos años son ellos quienes dirigen los rituales que permiten a la comunidad evolucionar y perpetuarse.

Palabras claves: baile, música, aprendizaje, ritual, niños, Ayacucho

\section{D'enfants à maîtres : I'initiation des artistes des danses rituelles d'Ayacucho}

\section{Résumé}

Les artistes des danses rituelles de compétition d'Ayacucho (Pérou) commencent jeunes voire parfois tout petits à apprendre leur art. Qu'ils veuillent devenir musiciens, danseurs de ciseaux, huaylías, pastores ou negritos de cinta, qu'ils vivent dans la Sierra ou à Lima, qu'ils apprennent d'une façon ou d'une autre, tous doivent non seulement apprendre à maîtriser les techniques instrumentales ou corporelles, mais plus que tout assimiler parfaitement les règles socioreligieuses ainsi que les us et

Encargada de cursos en el Instituto Nacional de Idiomas y Civilizaciones Orientales (rue des Grands Moulins, 75013 París, Francia).E-mail: jsaintsardos@hotmail.com 
coutumes. En effet, ce sont eux qui, après quelques années, dirigent les rituels qui permettent à la communauté d'évoluer et de se perpétuer.

Mots-clés : danse, musique, apprentissage, rituel, enfants, Ayacucho

\title{
From child to maestro: artists initiated into Ayacucho ritual dances
}

\begin{abstract}
The artists of Ayacucho ritual competition dances (Peru) begin learning their art when they are youngsters or even very small children. Whether they want to become scissor dancers, huaylías, pastores or negritos de cinta, whether they live in the Sierra or in Lima, whether they learn one way or another, not only do they all have to master instrument or body techniques but above all they have to assimilate socio-religious rules as well as habits and customs perfectly. Indeed after a few years' learning, they are the ones in charge of the rituals that enable the community to evolve and live on.
\end{abstract}

Key words: dance, music, learning, ritual, children, Ayacucho

En el departamento peruano de Ayacucho tres danzas son reservadas a bailarines especialistas, una característica que no se encuentra aparentemente en otros repertorios coreográficos de los Andes: la danza de las tijeras, de las huaylías y de los negritos de cinta. Bailarines y músicos de estas danzas tienen que iniciarse desde una edad temprana a su futuro oficio y después de poco tiempo estar en capacidad de cumplir un papel no solamente artístico sino también socioreligioso. Si algunos empiezan alrededor de los 15 años, muchos dan sus primeros pasos tan pronto pueden caminar. En pocos años, estos niños se convierten en personajes de suma importancia para el equilibrio de las relaciones socioreligiosas de las comunidades ayacuchanas y de sus migrantes. Esta responsabilidad precoz llama la atención: ¿cómo niños y jóvenes pueden dirigir estos rituales fundamentales para las comunidades? Intentaré contestar esta pregunta a través de la descripción del aprendizaje y de una reflexión sobre el estatus de estos niños iniciados.

\section{LAS DANZAS DE COMPETENCIA EN AYACUCHO Y LOS ACTORES DE TRANSMISIÓN}

\section{1. Las danzas}

La danza de las tijeras, la de las huaylías los negritos de cinta son todas danzas de competencia que comparten entre ellas varias características. En las tres se 
enfrentan dos grupos ${ }^{1}$ constituidos por un arpa, un violín y uno (cuando se trata de la danza de las tijeras) o varios bailarines (en el caso de las dos otras danzas)2 Durante varios días los bailarines se turnan en la pista de baile delimitada por el público en la plaza de armas y en otros lugares del pueblo y de sus alrededores. Demuestran diversas capacidades a través de una secuencia de piezas músicocoreográficas centradas en la agilidad de los pies, acrobacias, la resistencia al dolor o, entre otros, imitaciones teatrales. Cada una se basa en principios tácitos estrictos alrededor de los cuales los músicos y los bailarines tienen que variar e innovar (sobre este sistema músicocoreográfico complejo, cf. Saint-Sardos, 2011: 165-198).

Las tres danzas se bailan para fiestas que asocian eventos de los calendarios católico y agrícola, como por ejemplo la limpieza de las acequias (yarqa haspiy) y las fiestas de Santa Rosa de Lima y San Isidro Labrador en agosto en Andamarca3. Efectivamente, los artistas cultivan relaciones privilegiadas con las divinidades, ya sean católicas o andinas ${ }^{4}$, y aseguran el papel de mediadores entre ellas y la comunidad. La gente atribuía -y sigue atribuyendo, aunque menos - a los bailarines poderes sobrehumanos: dones de hechicería, adivinación y curación, a los que se añaden capacidades físicas fuera de lo común. Por lo tanto, los artistas son personajes a la vez admirados y temidos por sus paisanos. Los rituales que constituyen estas danzas son el corazón de las fiestas y tienen relaciones estrechas con los sistemas de $\operatorname{cargos}^{5}$ y reciprocidad (ayni). La emulación que provocan sus competencias permiten actualizar las relaciones socioreligiosas, es decir a la vez conservarlas y modificarlas según la situación del momento.

\section{2. Los transmisores}

La mayoría de los saberes necesarios para un futuro arpista, violinista o bailarín son transmitidos por los «maestros», los artistas consagrados. Actualmente, todos los maestros no han crecido en la Sierra y muchos han pasado y siguen pasando su vida entre la Sierra y Lima, la capital. Su participación como transmisor varía: es a la vez irregularmente reconocida y consciente según la forma del aprendizaje. Pero los artistas no son los únicos que transmiten saberes musicales a los aprendices. Los «capataces» contratados por los «cargontes»6 aseguran no solo la seguridad y el bienestar de los artistas en la medida en que los guían durante la fiesta. También

1 Existen competencias de más de dos grupos en unos cuantos pueblos por la estructura territorial y en Lima en nuevos contextos de estas danzas como los concursos.

2 Para la danza de los negritos de cinta, dos instrumentos musicales adicionales acompañan los bailarines: la tinya (un tambor con marco) y el espadín (un palo de metal).

3 Trabajo desde el principio de los años 2000 en el valle de Sondondo en el departamento de Lucanas.

4 Por falta de término más adecuado o apelación establecida, designo así las divinidades que no son católicas como los apu, los cerros.

5 Sistema de repartición de los gastos y responsabilidades de la fiesta.

6 Persona que asume un cargo, como pagar a los artistas. 
les explican las costumbres del pueblo: el desarrollo de la fiesta, el lugar de las ofrendas y la manera correcta de hacerlas para los más novatos. Desempeñan este papel particularmente en la primera oportunidad que un músico o bailarín llega a un pueblo. Las veces siguientes comunican su saber de manera menos formal, sobre todo a través de anécdotas o relatos de su experiencia.

Sin embargo, el resto de la población comparte muchos saberes de los artistas. Las personas mayores y las más experimentadas intervienen también en el aprendizaje aunque sea de manera informal. Sus comentarios enriquecen la experiencia todavía incipiente de los aprendices y completan la de los artistas mayores en las familias de artistas. Asimismo, las mujeres que no son artistas intervienen de manera significativa en calidad de esposa, hija, hermana de danzante y/o de músico. Como testigos cotidianos de la práctica de estas artes, pueden animar, aconsejar y orientar. Algunas indican y corrigen a su manera los errores de su hijo y ciertas madres llegan hasta dirigir el comienzo de la práctica. Esta tendencia concuerda con el papel que les incumbe en la familia pues son ellas las que crían a los niños. La mayoría son menos hostiles que sus esposos a que sus hijos escojan la carrera artística.

\section{3. Los aprendices}

En lo que se refiere a los aprendices, ellos deben ser originarios de la zona donde se practican las danzas, por lo menos por parte de uno de sus padres, y conocer el quechua. Estas capacidades lingüísticas no aparecen como una condición sine qua non, aunque los artistas que no hablan ni entienden el quechua son muy escasos hoy en día. Si no comprenden una parte de los intercambios orales y no pueden participar en estos, particularmente en los pueblos de la Sierra, estos artistas quedan marginados y no pueden asumir correctamente la parte social que implica su función. La procedencia social de los aprendices también es muy homogénea: la mayoría pertenecen a familias de artistas y todos vienen de un medio humilde. En cuanto a la edad de los aprendices, esta es variable. La mayoría de los danzantes de tijeras empiezan su aprendizaje entre los 12 y 15 años, y otros hacia los veinte. La edad de los músicos es ligeramente superior por el gasto importante que representa la compra del instrumento. Eso es distinto con los negritos, las huaylías y los pastores quienes aprenden casi todos desde muy niños acompañando a sus mayores. La inclinación de los niños o adolescentes hacia un instrumento parece altamente condicionada por el entorno. En las familias de artistas, la música y el baile forman parte de la vida diaria y la familiarización con el instrumento se hace a menudo progresivamente tocando y queriendo imitar a los mayores. A veces los padres alientan a su hijo o le pueden obligar también al principio. En otros casos se oponen y los niños aprenden a escondidas hasta el día en que, ya suficientemente avanzados para demostrar su motivación, revelan su secreto. Muchos de los negritos, huaylías y pastores ${ }^{7}$ simplemente siguen a

7 Son los hombres que bailan con las huaylías en la versión navideña de esta danza. 
sus padres o a los artistas mayores desde que caminan. En los casos a la vez de músicos y de bailarines, las nociones de placer y gusto aparecen en la trayectoria de los aprendices, incluso cuando empiezan a la fuerza. «Me gustó» se encuentra constantemente en los testimonios: un niño que no se apasiona por lo que toca o baila nunca podrá ser un maestro.

Sin embargo, para alcanzar el estatus de artista en estas tres danzas, es necesario cumplir otros requisitos. No puede ser artista quien quiere: casi todos pertenecen a especies de «dinastías». Sus padres, abuelos, tíos, hermanos, primos también son artistas. Muchas veces los mayores enseñan a los menores, o padres e hijos bailan y tocan juntos. Además uno no se improvisa artista: el aprendizaje de estas danzas resulta ser una verdadera y larga iniciación con la transmisión tanto de un saber estar como de un saber hacer. Por último solo el público puede consagrar a los aprendices, proponiéndoles contratos para fiestas importantes y en pueblos lejanos. Y las divinidades también los eligen a través de la ayuda que les bridan a cambio de sus ofrendas 8 .

Aprendices y artistas consagrados frecuentan una tercera categoría de personas: los «aficionados». Estos son particularmente numerosos en Lima, sobre todo debido a las evoluciones que afectan al sistema de transmisión y el complemento de recursos económicos que constituye la práctica de estos bailes. Los aprendices mayores que el público nunca ha reconocido como artistas ya no pueden beneficiar de este estatus por su edad demasiado avanzada ni tampoco pretender al estatus de bailarín o de músico. De vez en cuando, consiguen un contrato sin gran interés o siguen bailando o tocando como subalternos en las fiestas sin gran envergadura o por un sueldo irrisorio. Pero no todos estos aficionados tienen un nivel técnico mediocre. Sus carencias aparecen en campos más sutiles: sus capacidades creativas, su gracia (su «aire»), su conocimiento y respeto de las costumbres locales o su comportamiento. En realidad, la gran mayoría domina técnicamente el repertorio pero no manifiesta las aptitudes necesarias para llevar a cabo la integralidad del ritual, cuya competencia bailada constituye solamente el elemento central. La complejidad del rol de músico y bailarín de las danzas rituales no se limita a un conocimiento técnico de estos repertorios en la medida en que se tienen que dominar otras dimensiones.

\section{El contenido del aprendizaje}

Aunque las tres danzas rituales mencionadas sean repertorios de especialistas, estas se basan en el conocimiento colectivo previo a estos bailes. La posición de especialista ritual de los bailarines y de los músicos de estas danzas implica una pertenencia al grupo que practica estos rituales. Como miembros, comparten

8 El mejor ejemplo de esta elección es el dicho «pacto con el diablo» que los danzantes de tijeras decían hacer con la sirina (Saint-Sardos, 2011: 67-72) y cuyo fin trágico constituye el tema de La agonía de Rasu Niti de Arguedas (1962). 
saberes colectivos con los no especialistas, particularmente prácticas músicocoreográficas. Estos saberes se profundizan y pasan del campo de la práctica al de la performance. Los aprendices participaban hasta ese momento en el ritual como miembros de la comunidad pero no practicaban el lado músico-coreográfico. A partir del momento en el que se vuelven aprendices, se preparan para el papel de protagonistas de los elementos fundamentales del ritual que son las danzas de competencia. De conocedores se vuelven actores principales.

\section{1. La música y el baile}

Generalmente todos conocen las tonadas fijas 9 antes de empezar su aprendizaje por haberlas escuchado muchas veces durante las fiestas anteriormente. Al igual que los pasos del baile, el proceso de aprendizaje de los músicos consiste en una transposición técnica al instrumento y en mucha práctica. Para las tonadas que dejan más libertad y necesitan creatividad melódica, el proceso es diferente: en vez de aprender una técnica o una melodía, se trata de que el aprendiz comprenda la estructura y las características melódico-rítmicas profundas de estas tonadas. Este proceso complejo requiere más tiempo y una experiencia íntima del repertorio. Estas tonadas se aprenden después de las otras ya que el aprendiz tiene que desarrollar capacidades de análisis y creación además de las de imitación. Además, tienen que conocer los repertorios locales que se tocan para cada fiesta, lo que exige no solamente un aprendizaje técnico sino también una memorización del mismo repertorio cuando se trata de la costumbre de otro pueblo. Los futuros arpistas y violinistas tienen por supuesto que asimilar la técnica y ornamentación específica de su instrumento; pero tocar un instrumento requiere también otros saberes más prácticos. Hay que aprender a afinarlo, cambiarle las cuerdas 10 lo cual requiere tiempo, pues los aprendices primero tienen que adquirir una conciencia de la precisión de los sonidos así como de las capacidades físicas de los materiales que componen su instrumento.

En cuanto a los bailarines, estos tienen que aprender los pasos y la secuencia que los rige. Gran parte de estos aspectos ya han sido asimilados inconscientemente desde su niñez asistiendo a las danzas puesto que representan rituales comunitarios. Toda la población conoce los pasos y las tonadas, y puede criticar si no se respetan las reglas. Así, el aprendizaje y la especialización consiste en un refuerzo de estos saberes y en un trabajo técnico en los diferentes pasos. Sin embargo, algunos pasos de la danza de las tijeras no benefician de ninguna práctica preparatoria: son los que comprueban la resistencia al dolor (pasta) y la acrobática bajada de la torre de la iglesia en una cuerda (torre bajay). Peligrosos y dolorosos, estos se ejecutan solamente para las fiestas, como lo explicaba el aprendiz Lizandro Villegas Alarcón «Paccha Ccapari» (23 años):

9 Llamo así a las tonadas cuya melodía es definida y no debe ser creada por los músicos.

10 Hasta hace poco los arpistas tenían que fabricar ellos mismos las cuerdas de tripas. 
En la casa practicas, te duele pues cualquier cosa. Pero cuando estás en la plaza, en plena competencia, no te duele nada pues. Creo que por la música, creo que algo así...

Asimismo, la preparación de estos pasos no solo implica la experiencia física porque la situación de performance permite ella sola su realización. Aparte de las facultades ligadas a lo físico y coreográfico de las danzas, las huaylías, los pastores y los negritos se inician al arte oratorio y a la composición de cantos, indispensables para el ejercicio de sus funciones de bailarines. Además, los danzantes de tijeras y los pastores (en menor medida) deben también hacer esfuerzos particulares para acompañar su pasos con sus metalófonos (las tijeras o la sonaja).

\section{2. Los códigos sociales y las costumbres}

Gran parte del aprendizaje consta de la adquisición de prácticas no musicales sino sociales y rituales. Al igual que los saberes musicales, la mayoría de estas no son comunes solamente a los artistas sino a un grupo más amplio. Los músicos y bailarines de las danzas de competencia se destacan de los demás por su conocimiento diverso y profundo de los usos y de las costumbres. Empezando con las relaciones familiares: de las relaciones con los padres para los menores a las con la familia que los mayores están en edad de fundar, estos lazos son muy valorados en el aprendizaje. El comportamiento familiar de los aprendices queda anclado en los valores cultivados en la Sierra: la familia en su sentido amplio y la familia ritual sirven de unidad social de base. En Lima, estos modelos adquieren

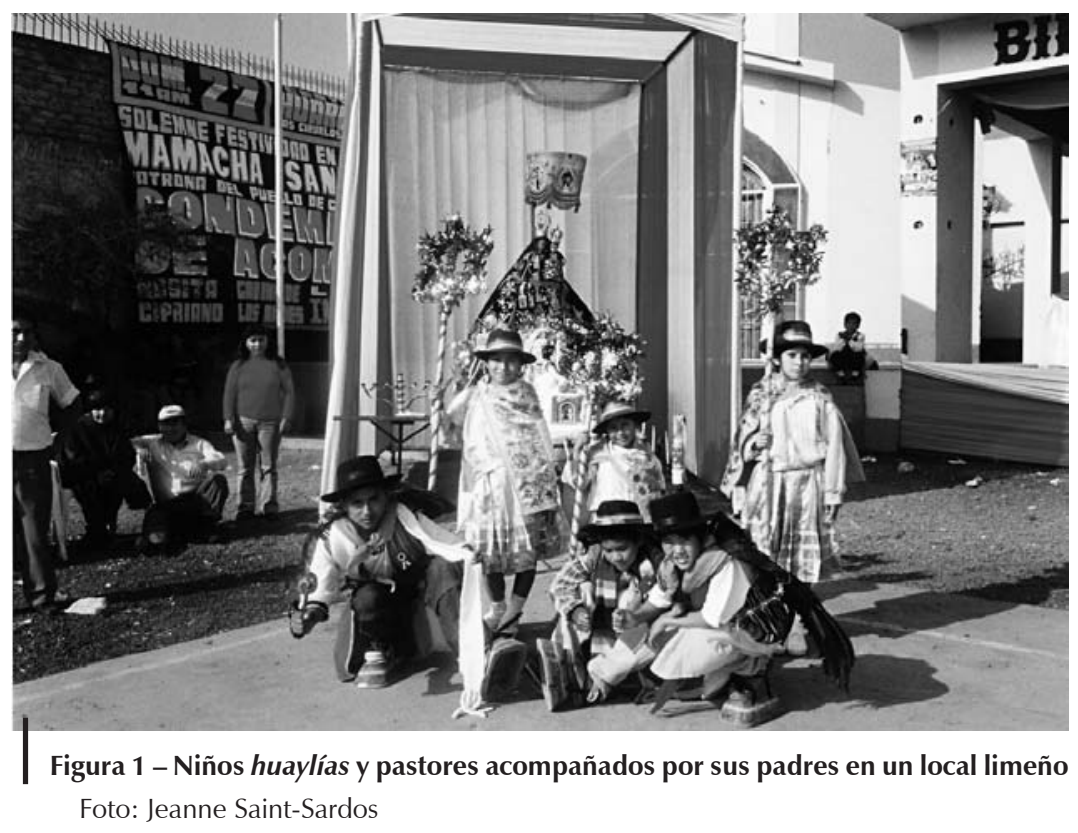


tanto más peso cuanto que las familias están divididas por recorridos migratorios. Los aprendices pueden encontrar en esta relación artística referencias de las cuales han carecido en su vida personal. La importancia de los lazos familiares no representa solamente un valor común particularmente sustentado por los artistas sino un verdadero eje social alrededor del cual se organiza la transmisión.

Paralelamente a la valorización de las relaciones familiares, otro comportamiento es inculcado en el medio artístico: el respecto a los mayores. Por cierto, este es esencial en el proceso de transmisión para ser aceptado por un maestro o referente, y de forma general por el medio artístico. Se aplica también a las relaciones del artista con sus pares, y al momento de enfrentarse con un adversario mayor, no lo desacredita como lo haría con un contemporáneo suyo. Aparte de las reglas sociales que rigen el medio ayacuchano, los artistas aprendices deben también aprender las costumbres de cada pueblo. Ello depende en parte de la forma de aprendizaje y se realiza de diferentes maneras: por inmersión desde la infancia, siguiendo a los maestros, o por los relatos de sus mayores o de personas de confianza originarias de estos pueblos. Como artistas es necesario que participen también a los repertorios músico-coreográficos comunitarios conexos en las fiestas. Los músicos conservan ahí su papel de especialistas y deben tener en su repertorio danzas y músicas estacionales locales. Los bailarines no tienen esta obligación pero deben participar aunque sea de manera torpe. Así las huaylías generalmente conocen bastante huaynos que cantan para divertir o hacer bailar a la concurrencia durante los momentos de descanso. El dominio de los otros repertorios locales es menos común en los otros bailarines pero es muy apreciado por el público.

Por lo tanto cada primer contrato en un lugar es de suma importancia porque es muchas veces la primera inmersión directa con estas costumbres. Los jóvenes artistas tienen que estar muy atentos a todas las indicaciones que la gente le pueda dar y siempre tener en mente las informaciones que cosechan sobre las características y costumbres locales. Su transgresión les puede causar perjuicios en su carrera artística, lo que puede ocasionar la pérdida de contratos y detener su crecimiento profesional, dejándolos definitivamente en el estatus de aficionados en los casos los más graves.

\section{3. Los rituales}

Además del ritual central que constituye el baile, los aprendices van aprendiendo otros rituales conexos. Se trata de ofrendas para las divinidades andinas y la plaza11. Queda muy difícil tratar el tema pues los artistas no dejan participar a otras personas excepto su capataz. Según los testimonios, siempre es el mayor o el más experimentado que dirige las ofrendas y los más jóvenes van aprendiendo observando e imitándole. Los discursos de los maestros y de los capataces

11 Los dones de hechicería y curación que muchos artistas decían tener antaño parecen haber desaparecido de la práctica. 
refuerzan las observaciones y explicitan ciertas acciones, aunque la asimilación de los rituales queda una cuestión de imitación.

\section{MODOS Y TÉCNICAS DE TRANSMISIÓN}

Antes de volverse artistas, bailarines y músicos tienen que pasar por un largo aprendizaje. Este proceso no es idéntico para todos y puede tomar tres formas diferentes según la función a la cual aspira el aprendiz y las circunstancias en las cuales empieza. Así los futuros dansaq (danzante de tijeras en quechua) y músicos aprenden con un maestro o solos, en cambio las huaylías y los negritos de cinta se integran a un grupo desde el principio.

\section{1. Aprender con un maestro}

El aprendizaje con un maestro se realiza sobre la base de un intercambio económico: el maestro forma el aprendiz y en cambio el aprendiz trabaja por él. El niño (o el joven) sale a vivir a la casa de su maestro si no vive en el mismo pueblo, participa en las tareas domésticas, trabaja en la chacra y cuida a los animales como cualquier miembro de la familia. Cuando el maestro tiene un contrato en un pueblo, el aprendiz lo acompaña. Ahí lo ayuda, lo protege, y a cambio el maestro le permite participar en la fiesta y encontrar a otros artistas. Además, el aprendiz se hace conocer poco a poco y las fiestas lo ayudan a veces a demostrar algunos de sus talentos, especialmente en el caso de que algún problema le suceda a su maestro. En este caso lo puede remplazar y conseguir así su primer contrato. Pero un maestro no acepta a cualquiera. Se muestra más propenso a formar a un miembro de su familia o a una persona cercana porque un extraño puede hacerle sombra a su hijo o a su sobrino. La prioridad se da a la familia. Sin embargo, el inicio del aprendizaje confirma o no la decisión del maestro: si el discípulo demuestra las capacidades necesarias, lo mantiene como tal, en caso que no, lo devuelve a sus padres.

Generalmente el aprendiz se inicia con su maestro después de un tiempo de práctica solitaria y más bien lúdica según los testimonios. Solo o con otros niños, imita a los dansaq y músicos durante la época de la fiesta; forman grupos, se dan nombres de artistas y silban para acompañar sus pasos. Pero después de estos juegos, son escasos los que comienzan realmente su aprendizaje. Además, antes de buscar a un maestro, el futuro aprendiz de músico ya ha tenido un contacto seguido con el instrumento — que pertenezca a un miembro de su familia o que aproveche fiestas o encuentros para utilizar los instrumentos de los músicos- y ya ha intentado reproducir melodías de vez en cuando.

La música de la danza de las tijeras constituye el primer repertorio de los arpistas. Su base no es de la más difícil y permite ejercitar las manos dando fundamentos técnicos para lograr los demás repertorios. El joven va desarrollando su creatividad y aprende a inventar así como adaptar melodías para las tonadas que tienen partes 
libres. Todo este proceso necesita entre dos y cuatro años y pocos empiezan antes de la edad de 15 años.

Con respeto al aprendiz de dansaq, una vez encomendado por sus padres a un maestro, aprende a manejar las tijeras. Esto dura algunas semanas antes de que el maestro le enseñe el baile que el alumno debe practicar tocando las tijeras y silbando, o a veces al sonido del violín y arpa. Si todos estiman que nunca terminan de aprender y mejorarse en el baile, se inician como verdaderos danzantes cuando logran un primer contrato en un pueblo después de un aprendizaje elemental de unos 5 años. Ejecutan entonces la secuencia completa y participan a la competencia en la plaza por primera vez. A los pasos ensayados, numerosas veces añaden faquirismo y acrobacias que realizan únicamente en esta situación de performance, es decir bajo la presión de todo el pueblo y en un contexto ritual particular. Si durante el aprendizaje el maestro se da cuenta que el alumno no tiene las capacidades necesarias, lo devuelve a sus padres. Si, por lo contrario, está satisfecho con él, lo considera como su discípulo de allí en adelante. Pero eso significa también que el aprendiz tiene que volverse poco a poco independiente, aunque siempre puede pedir consejo al que sigue siendo su maestro. No retiene de su maestro solo un saber técnico sino una experiencia de vida. Después del fin del aprendizaje, conserva relaciones especiales con él. No le considera como un simple profesor sino más bien como a un padre. Este lo aconseja, lo guía en su vida y su opinión tiene mucha importancia. Su discípulo es su sucesor y de cierta manera le representa. Por eso tiene que dar una imagen correcta en todos los aspectos para ser digno de la reputación de su maestro. Este lo introduce también al medio de los artistas, lo cual constituye una gran ventaja; siguiendo a su maestro el discípulo da a conocer su nombre y sus capacidades para ir integrándose al medio de los artistas.

\section{2. Aprender solo}

Antaño en la Sierra, el maestro era un personaje casi ineludible. Pero la evolución de las costumbres, la movilidad creciente de la población y la migración hacia la capital hicieron intensificarse el aprendizaje en solitario que concierne actualmente a la mitad de los aprendices, tanto en la Costa como en los pueblos andinos.

No tener maestro implica estar al acecho de cualquier transmisor, lo cual puede multiplicar los puntos de referencia. El aprendiz recupera un elemento de un maestro, entiende una técnica observando un segundo e intenta imitar el estilo de un tercero... Cualquier músico o danzante se vuelve entonces un transmisor potencial a quien el joven observa con una intensidad proporcional a la fama del artista. No sería exacto decir que un discípulo tiene como modelo solamente a su maestro, aunque este sigue siendo su principal referencia. Le enseña la técnica y un repertorio que le permite volverse independiente y, gracias a sus críticas, el aprendiz va adquiriendo un sentido crítico. Si generalmente conserva el estilo de su maestro, se forja el suyo observando a otros maestros y adueñándose de los elementos que le gustan y que le parecen conformes con lo que se le ha 
enseñado. El que aprende asimila de esta manera desde el principio pero tiene que desarrollar también capacidades críticas así como su propio estilo con el fin de hacer coherente un conjunto de elementos que vienen de todas partes y que carece de unidad. La mayor diferencia consiste en el parámetro de la distancia: el aprendiz no reproduce un ejemplo de su maestro, destinado a él, repetido y que observa de muy cerca. Se contenta con las escasas ocasiones en las cuales puede frecuentar a los maestros. Además, la relación entre el discípulo y su maestro los hace cercanos en lo afectivo y técnico, especialmente en lo que concierne al estilo. Ahora bien, esto no se encuentra en la relación que el que aprende solo pueda mantener con uno de los músicos o danzantes que le sirven de modelo. Sucede muchas veces que el joven no los conoce personalmente. Sin embargo, el acercamiento a estos referentes y a sus consejos perspicaces son esenciales para que el aspirante a danzante o a músico logre su meta.

Por otro lado, el aprendizaje varía en cuanto a la duración de la adquisición de los saberes. Suele ser más largo sin maestro porque el aprendiz no tiene a nadie quien lo guie y le indique el buen camino. Desde el inicio hasta el fin del proceso, el aprendiz dirige su avance, siembra y cosecha los frutos de su labor. Por supuesto el joven danzante o músico no debe nada a ningún maestro en la medida en que nadie se encarga de su aprendizaje. Para agradecer por un consejo o una ayuda, invita al danzante o al músico algo que tomar, le hace un favor a cambio pero no llega a trabajar para él. El orden de asimilación del repertorio depende esencialmente del gusto del aprendiz de músico y la mayoría empieza por la danza de las tijeras. Sin la influencia de un maestro, se abre más a ciertas modificaciones de la técnica. Pero esta libertad se gana a costa de numerosos esfuerzos.

Aprender sin maestro significa también avanzar sin consejos ni críticas, y los que proceden así son conscientes de los inconvenientes de esta ausencia. Más allá del simple saber técnico, es la experiencia de danzante o músico que les hace falta. El problema de la integración al medio de los artistas se vuelve crucial desde que el aprendiz es capaz de asumir sus primeros contratos. Sin fama, nadie le propone contratos interesantes. Su inicio en el medio artístico es difícil, sin ayuda puede sobresalir solamente gracias a sus capacidades. Debe relacionarse con uno o varios maestros, enseñarles su potencial para que reconozcan sus capacidades y le den algunos consejos. Esto le permite tener la crítica exterior de la que carecen. Debe convencerlos para que lo lleven con ellos a presentaciones no pagadas o para pequeños contratos. Esta última etapa le permite hacerse conocer; es justo durante esta época que la mayoría recibe su «chapa», su nombre de artista definitivo. Estos aprendices sin maestro compensan también los inconvenientes de esta forma de aprendizaje por una gran motivación y una práctica sin descanso. Si quieren hacerse aceptar y volverse maestros un día, tienen que ser por lo menos intachables en el campo técnico. Al contrario de los que tienen un maestro o una familia de artistas, deben su éxito solamente a su forma de tocar o bailar y no se pueden permitir ninguna negligencia en este campo. 


\section{3. Aprender en grupo}

Para las huaylías, los pastores y los negritos, el aprendizaje toma otra forma en la medida en que se inician en un grupo y desde su infancia, es decir antes de los 10 años. Siguen los pasos de los miembros de su familia cercana que bailan, muchas veces desde que se mantienen de pie y dan sus primeros pasos. Se ponen a bailar de la misma manera que acompañan e imitan a sus padres y a sus mayores en las actividades de la vida cotidiana. Suelen tomar sitio al costado de los más jóvenes pero sin dirigirse de una forma especial: andan libremente en la pista de baile sin seguir a los demás. El paso del mimetismo infantil a la integración a un grupo de bailarines como miembro de pleno derecho se hace en total continuidad. Los niños que no pertenecen a una familia de artistas empiezan a una edad un poco más avanzada. Al igual que los jóvenes dansaq y músicos, intentan imitar a los maestros desde muy pequeños. Buscan desde temprana edad ocasiones para acompañar a los mayores y progresivamente relacionarse con una persona o un grupo. A menudo, la intervención de los padres es determinante, ya sea para introducirlos al medio durante las fiestas o para entregarles a bailarines experimentados.

Este modo de aprendizaje combina las características de los dos otros: en ciertos aspectos se asemeja al aprendizaje con un maestro, y en otros, a la práctica en solitario. El niño es guiado y se encuentra bajo la autoridad de una o varias personas a quienes considera como sus maestros. Una vez aceptado en el grupo, los primeros bailarines lo aconsejan y corrigen hasta que lo puedan ascender como segundo bailarín. Además se benefician de varios referentes desde el principio. La dependencia hacia los mayores es menos fuerte en la medida en que se reparte entre varias personas y que no viven juntos, excepto que los referentes sean sus padres. Esto no impide que la relación con ellos sea muy fuerte, pero es diferente, en parte por la edad menos elevada de los aprendices. La ventaja de esta situación consiste en el ambiente competitivo que reina generalmente entre los aprendices: todos quieren ser el que mejor baila, al quien el caporal o la guiadora llevará a tal pueblo, el que tomará el sitio del segundo bailarín en caso de ausencia, el que tendrá primero su primer contrato... Estas pequeñas rivalidades raras veces tienen efectos negativos pero constituyen más bien una emulación que prepara a los niños al espíritu de competencia que domina en estas danzas. Asimismo los aprendices encuentran en este tipo de aprendizaje un entorno tranquilizador mientras se enfrentan progresivamente a las dificultades que los esperan. Son ellos los que tienen que solicitar a los mayores e imponerse poco a poco como un miembro del grupo.

Una diferencia esencial entre este modo de aprendizaje y los otros dos radica en el hecho de que la situación de performance no representa el objetivo del aprendiz sino uno de los contextos comunes de la práctica. Al principio muchos niños se juntan a un grupo solamente para las performances. Nadie espera de ellos habilidades particulares; solamente siguen a los demás como pueden. Cuando empiezan a dominar pasos simples, compiten durante las fiestas cuando se presenta tiempo libre y un adversario de su edad en el grupo del frente. A medida 
que van creciendo, las competencias son más frecuentes y largas, con pasos más numerosos. Así adquieren la experiencia de la performance directamente, sin preparación y no por procuración; mejoran en gran parte gracias a esta experiencia mucho antes de lograr el estatus de artista consagrado.

\section{4. Las técnicas de aprendizaje}

Las técnicas de aprendizaje no se pueden estudiar fácilmente porque la enseñanza es poco verbalizada y es difícil conseguir informaciones sobre esta actividad. Fuera de algunas excepciones, las técnicas parecen poco elaboradas, y generalmente a cargo del aprendiz en la medida en que el maestro modifica poco su comportamiento comparado a su práctica solitaria. El aprendiz adquiere gran parte de la técnica por mimetismo, a raíz de largos periodos de observación. Cuando toma el ejemplo de varios artistas, la asimilación procede de una imitación más pensada y más estructurada que le permite remediar a la imprecisión de la memorización en una situación más difícil que el cara a cara con un maestro.

Asimilar no lo es todo: al igual que los artistas experimentados, el aprendiz ocupa gran parte de su tiempo ensayando. En cierto modo pasa de lo abstracto a lo concreto practicando las técnicas que ha observado. Trabaja generalmente sobre una pieza que repite varias veces; esto le permite probar varias técnicas, ideas de realización y maneras de tocar o bailar una pieza. Además, esto lo prepara físicamente; la necesidad de este trabajo aparece en la mayoría de los discursos,

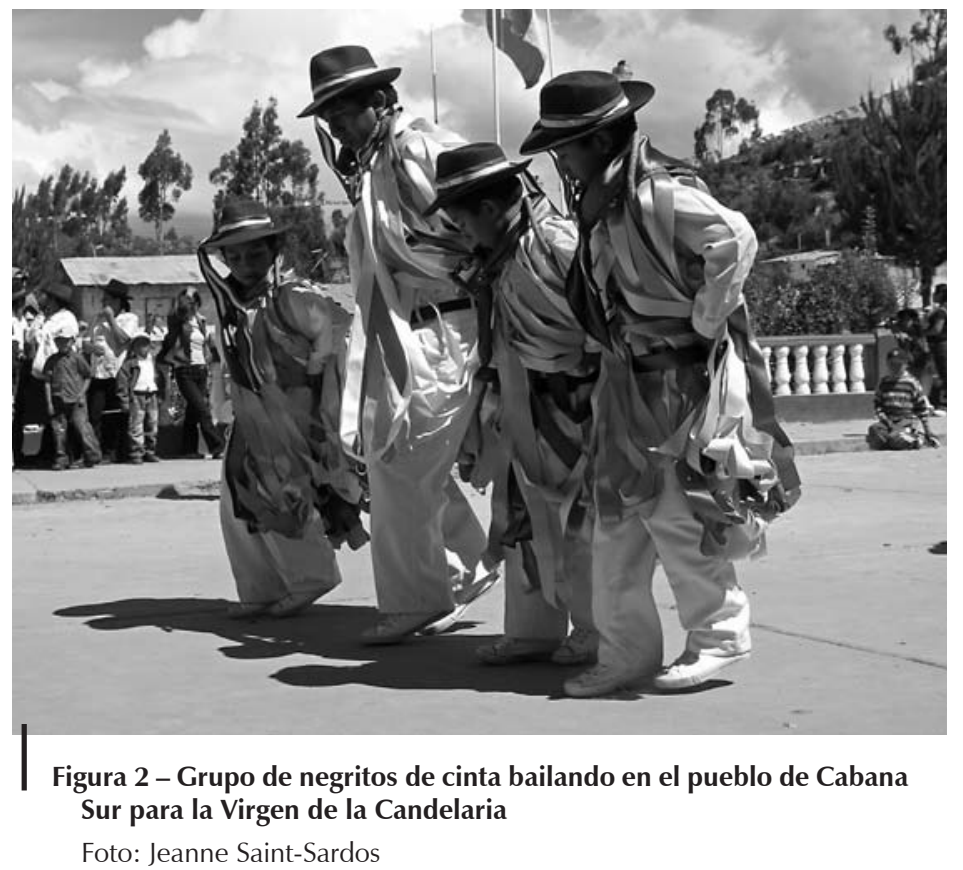


y sobre todo en el medio urbano. Dicen tocar o bailar cada vez que pueden, cuando tienen un rato libre durante el día, entre sus actividades cotidianas. Conviene mencionar también una técnica más precisa utilizada por los bailarines: la repetición de un elemento peculiar. Esto sirve para adquirir el dominio técnico y físico de un punto preciso con el fin de integrarlo a un conjunto coherente. Los músicos recurren muy poco a esto y prefieren repetir tonadas enteras. La noción de ornamento existe porque algunos tienen un nombre pero nunca se tocan aislados de la tonada. Los ornamentos no se practican ni se enseñan aparte sino en una secuencia estructuralmente coherente de la tonada. Un encadenamiento difícil de unas notas se adquiere repitiendo la tonada entera. Si los músicos no desprecian el aspecto técnico, muy al contrario, no lo separan de las melodías.

El habla participa poco al proceso de aprendizaje y la verbalización de la técnica (así como de la estructura musical) es muy reducida. Excepto unos términos musicales occidentales, particularmente para los modos y las notas, el lenguaje empleado utiliza palabras comunes, simples y no propias de un dominio. No existe realmente un vocabulario específico para la música o el baile: hasta el nombre de las piezas músico-coreográficas evocan cosas concretas (ej.: waychaw —una especie de pajarito—, torre bajay — la bajada de la torre-) o onomatopeyas (ej.: tipaq tipaq). Non obstante, para la transmisión de la experiencia personal, el habla se impone como único vector en la mayoría de los casos. En quechua o en español, en la Sierra o en Lima, las anécdotas y los cuentos conservan su importancia. Que se dirijan a sus discípulos o a los demás, los músicos y bailarines aportan su experiencia de la vida. Asimismo, gracias a estos relatos, el aprendiz no se construye solamente como técnico sino como persona y artista.

Ahora, con la influencia de la vida limeña, otros medios de enseñanza se incorporan. Así la escritura musical suscita un interés creciente entre los músicos pero no se utiliza, por lo menos bajo su forma occidental; el pentagrama (y lo que transmite) no responde a las exigencias de los músicos y de lo que quieren transcribir. Los maestros de Lima reflexionan también sobre los métodos de enseñanza de la técnica y la manera de transmitir su arte a los jóvenes hijos de migrantes. Por eso utilizan técnicas sonoras y audiovisuales actuales de la vida cotidiana. Casetes, CD, DVD y videos por internet están ahora al alcance de los ayacuchanos, que sean migrantes o no. Estos permiten grabar las fiestas para luego mirar o escucharlas a voluntad y memorizar las diferentes piezas, observar novedades y criticar a los otros o a sí mismo. Estos soportes audiovisuales se vuelven puntos de referencia suplementarios que estimulan la creatividad personal de los aprendices y de los artistas en general. Existe también una preocupación pedagógica que se concreta en la elaboración de ejercicios y en una atención diferente con el aprendiz. El maestro intenta entender las necesidades de sus aprendices y adapta su enseñanza a las mismas. Por ejemplo, puede crear ejercicios técnicos simples fundados en la repetición de un elemento problemático. Una actitud semejante es innovadora con respecto a la enseñanza tradicional y parece ganar terreno en Lima. Por supuesto la existencia de diversas formas de enseñanza en la capital no es ajena a esto. 


\section{LOS PODERES DE LOS NIÑOS ESPECIALISTAS RITUALES}

\section{1. ¿Niños iniciados?}

Como lo muestra esta descripción, la enseñanza de estas danzas sobrepasa un simple aprendizaje técnico del baile o de la música. Los aprendices se vuelven expertos no solo en su arte pero también en los campos social y religioso. Si otros miembros de su comunidad comparten algunos de sus saberes, son los únicos que juntan todas estas capacidades. Y más que todo, se incorporan a un grupo social particular, el de los artistas rituales. Si no existe realmente ritos de paso, tal como los describe Van Gennep (1909), que señalan este cambio, este aprendizaje constituye una verdadera iniciación. Efectivamente se trata de «un rito de formación discontinua e irreversible del individuo como representante de una categoría social cuyo atributo esencial es la experiencia común y transitiva de esta transformación puramente cultural» (Zempleni, 2004: 375). Esto se refleja en el testimonio de un danzante de tijeras12: «[el aprendiz] absorba toda su experiencia del maestro». Este comparte con su discípulo lo que recibió de su propio maestro, a lo cual se añade su práctica personal. Las relaciones entre maestros y aprendices, calcadas en las relaciones familiares, refuerzan esta idea: la experiencia compartida pasa de generación en generación de artistas como una herencia.

Sin embargo, si este proceso posee un carácter iniciático indudable, el hecho de perder el estatus de aprendiz por empezar a encabezar los rituales no significa la adquisición de un estatus de iniciado. Ocurre cuando los dansaq y los músicos consiguen su primer contrato y cuando las huaylías, los pastores y los negritos de cinta empiezan a ocupar el sitio del segundo bailarín en los grupos, por tomar las decisiones y componer los cantos colectivos con el primer bailarín. Desde ahí, son considerados como verdaderos artistas pero todavía nadie les atribuye la denominación de «maestro». Esta aparece solamente cuando se encuentran en la edad de poder transmitir su experiencia, es decir paradójicamente cuando la mayoría de los bailarines ya no «están de moda» y ya no tienen contratos importantes. El círculo debe cerrarse:

solo volviéndose iniciador, uno se vuelve plenamente un iniciado (Zempleni, 2004: 377).

Así, de manera muy sorprendente, todos estos artistas dirigen los rituales antes de terminar su iniciación.

\section{2. Una ambigüedad necesaria}

Si la madurez iniciática de los artistas de estas danzas rituales no aparece como necesaria, la madurez social no lo es tampoco: en las comunidades ayacuchanas

\footnotetext{
12 Juan Díaz Flores, «Chino de Andamarca», 35 años, 2003.
} 
un joven ingresa a la categoría de adulto casándose o teniendo hijos. Pero es precisamente a la edad aproximativa de los 25 años —o en estas condicionesque la gran mayoría de los bailarines dejan de practicar su arte. Los papeles de dansaq y de huaylía están ligados a cierta inmadurez social. Este caso no se aplica a los otros bailarines y músicos que continúan bailando y tocando, muchas veces hasta una edad avanzada.

La falta de experiencia de parte de estos artistas se compensa por un largo y exhaustivo aprendizaje. Esta oposición les confiere una carácter ambiguo entre el profundo respeto y una posible transgresión de los usos sociales. Esta ambigüedad se expresa también en la música y el baile con artistas que prueban los límites músico-coreográficos y físicos intentando superarlos sin transgresión (Saint-Sardos, 2011). Hasta los grupos no son homogéneos en cuanto a la edad de los artistas: en la mayoría de los casos se mezclan todos tipos de edades. La cohabitación de estas nociones de experiencia, de orden y de transgresión ofrece un cuadro adaptado a la revisión de las relaciones sociales. Deja posibilidades de cambio sin llegar al desorden de las fiestas como el carnaval (Albert-Llorca, 2002). Permite a la vez la conservación y la evolución, y entonces la actualización de las relaciones sociales. Efectivamente las competencias bailadas son solamente la parte visible de competencias sociales más profundas.

Además, estas características son particularmente interesantes en el contexto de migración que conoce la comunidad ayacuchana. Con el éxodo rural y la violencia del terrorismo durante los años 1980 y 1990, muchos ayacuchanos se instalaron en Lima y cultivan ahí su identidad a través de numerosas costumbres. Por eso ahora estas tres danzas rituales se desarrollan también en la capital (Bigenho, 1993; Nuñez, 1983; Saint-Sardos, 2011). La comunidad de los migrantes ayacuchanos necesita a la vez un cuadro para construirse en su nuevo medio pero también una adaptabilidad frente al contexto multicultural de la capital. Por cierto, la edad de los artistas y su gran conocimiento de las reglas sociales facilitan esto. Por otra parte, los artistas encuentran en el medio multicultural de Lima una fuente de inspiración para alimentar la creatividad que les pide la estructura misma de las tres danzas. Muy lejos de las comunidades serranas, estos niños y jóvenes herederos de un conocimiento profundo del funcionamiento de la sociedad hacen crecer estas tres danzas en Lima, en gran parte gracias al carácter antinómico de sus personajes.

\section{Referencias citadas}

ALBERT-LLORCA, M., 2002 - Regards anthropologiques sur la fête. Parcours, 25-26: 345355.

ARGUEDAS, J. M., 1962 - La agonía de Rasu Niti, 24 pp.; Lima: Icaro.

BIGENHO, M., 1993 - El baile de los negritos y la danza de las tijeras: un manejo de contradicciones. In: Musica, danzas y máscaras en los Andes (R. Romero, ed.): 219251; Lima: Pontificia Universidad Católica del Perú (PUCP)-Instituto Riva-Agüero. 
De niños a maestros: la iniciación de los artistas de las danzas rituales ayacuchanas

NUÑEZ REBAZA, L., 1983 - La vigencia de la danza de las tijeras en Lima metropolitana, 448 pp.; Lima: Asociación peruana para el fomento de las ciencias sociales (FOMCIENCIAS).

SAINT-SARDOS, J., 2011 - S'affronter pour mieux unir : danseurs et musiciens de trois danses d’Ayacucho (Pérou), 396 pp.; París: Université Paris-Sorbonne. Tesis de doctorado. http://www.e-sorbonne.fr/theses/s-affronter-mieux-unir-danseurs-musiciens-troisdanses-d-ayacucho-perou-0

VAN GENNEP, A., 1909 - Les rites de passage, 288 pp.; París: Nourry.

ZEMPLENI, A., 2004 - Initiation. In: Dictionnaire de l'ethnologie et de l'anthropologie (P. Bonte \& M. Izard, eds.); 375-377; París: Presses Universitaires de France (PUF). 